\title{
ACCELERATING GLASSY RELAXATION IN THE FRENKEL-KONTOROVA MODEL
}

\author{
Shelly Shumway \\ Argonne National Laboratory, Bldg 223; Argonne, IL 60439 \\ James P. Sethna \\ Laboratory of Atomic and Solid State Physics \\ Cornell University; Ithaca, New York 14853-2501
}

\begin{abstract}
We have developed a method for accelerating equilibration in numerical simulations of glassy models, using the Frenkel-Kontorova model for developing and testing the algorithm. This simple model is like a glass in that relaxation times diverge rapidly as the critical temperature is reached, so that no matter how slowly it is cooled, the system eventually gets stuck in some random metastable configuration. By adding "numerical enzymes," or long-range Monte Carlo moves which precisely eliminate certain barriers to relaxation, we can equilibrate rapidly to significantly lower temperatures. Our numerical method for developing these enzymes is a learning algorithm motivated by Darwinian evolution.
\end{abstract}

We begin this paper with a brief discussion of the Frenkel-Kontorova [FK] model, which is the simplest we know of that includes incommensurability and frustration in a natural way. We then discuss a general approach for equilibrating numerical models of, e.g., configurational glasses, in which the Hamiltonian is simple and not random, but relaxation time scales are so slow that the system always gets stuck in high-energy, random configurations. We find that "numerical enzymes", or complicated multiple-atom Monte Carlo moves fine-tuned to bypass the barriers to relaxation, can be of great assistance for shedding light on the underlying behavior of a model with natural dynamics too slow for effective numerical study using normal techniques. Although such enzymes may be subtle and complex, a learning algorithm motivated by principles of Darwinian evolution and natural selection can find them.

The FK model consists of a one-dimensional chain of atoms, each connected to its nearest neighbors by springs, with an externally applied sinusoidal potential:

$$
H=\frac{K}{2} \sum_{j}\left(x_{j+1}-x_{j}-\alpha\right)^{2}-V \sum_{j} \cos 2 \pi x_{j} .
$$

Frustration occurs when the periodicity of the applied potential competes with the tendency of the springs to keep the atoms evenly spaced; we work in the "pinned" limit, ${ }^{1}$ in which the spring constant $K$ is small. We have investigated ${ }^{2}$ the behavior on cooling from a finite temperature, at which atoms undergo thermal motion, to a zero temperature frozen chain. Any finite cooling rate $\gamma$ is too fast for the system to be able to equilibrate all the way into the ground state, although cooling more slowly does result in somewhat better equilibration. 


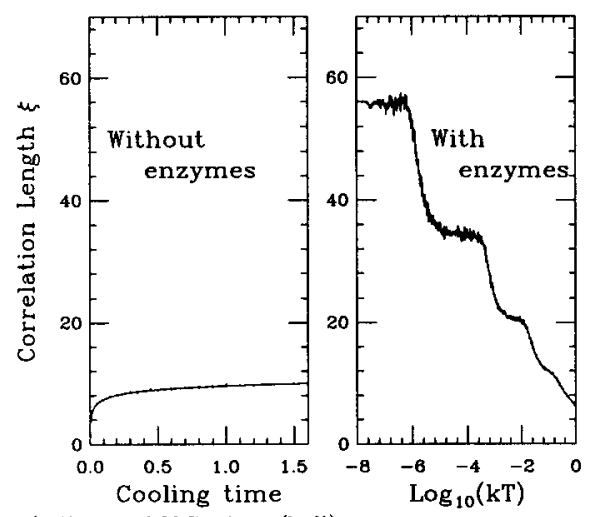

(Millions of M.C. steps/ball)

Figure 1: Dynamical Behavior of the FK Model. Without enzymes, no significant ordering develops even with very slow cooling. The addition of enzymes allows the true underlying behavior to be explored numerically in reasonable computer time. $(\mathrm{K}=1, \mathrm{~V}=3$, lattice spacing $=1)$
We have run simulations of this model using a standard Metropolis Monte Carlo algorithm, and have found that the correlation length $\xi$, which measures the average length scale of regions in local ground state configurations, diverges incredibly weakly as a function of cooling time $1 / \gamma$ :

$$
\xi \sim \log \left(\log \left(\frac{1}{\gamma}\right)\right)
$$

as shown in Figure 1 and discussed in detail in reference [2]. If normal simulations had been the only way to study the model, we would probably not even have suspected that the limiting behavior was actually an ordered ground state. This model is glassy in the sense that diverging time scales cause the "melt" to fall out of equilibrium at some historydependent temperature and get stuck in one of many possible metastable configurations, generally exhibiting no longrange order. Like a glass, cooling a lot slower allows equilibration to only a slightly lower temperature.

Widom, Strandburg and Swendsen ${ }^{3}$ encountered a similar situation in simulating a two-component Lennard-Jones [LJ] system. They, like others, found its dynamical behavior to be glassy. By adding special three-atom moves and long hops to the normal small relaxations in Monte Carlo simulations, however, they were able to equilibrate much faster and thus discover underlying quasicrystalline behavior in a system with suitable LJ parameters.

Motivated by their work, we have set out to develop a general method for finding special moves to accelerate equilibration and thereby illuminate the underlying behavior of various glassy models. We have started with the FK model because of its simplicity, but hope to be able to apply the technique to more realistic models whose true equilibrium behavior is obscured by complexity and diverging time scales in simulations.

The "numerical enzymes" we are looking for must be able to provide an accelerated mechanism for relaxation of metastable states. Calculating them directly will generally be impossible; we need to be able to find them with out knowing in advance what the high-energy configurations or corresponding lowenergy relaxed states are like. We have therefore developed a learning algorithm to get the computer to systematically discover and fine-tune effective enzymes with only minimal guidance. The method works well in the FK model.

Our approach, like the genetic algorithms that have been used with great success by engineers and others, ${ }^{4}$ is motivated by biological principles. The basic idea is that the solution to a problem is discovered through evolution of a population of some kind, in which an appropriate formula for health or fitness determines each individual member's survival and reproduction. Those that do 
poorly die off, and are replaced most often by the offspring of exceptionally healthy individuals. In our algorithm, we have a population of moves: each individual is represented by an $n$-dimensional vector telling how far to attempt to move each of $n$ atoms. We use a modified Monte Carlo-like code in which we select one individual at random from this finite population of discrete moves for every update of the FK chain, keeping track of each one's performance in promoting equilibration. Detailed balance is preserved by multiplying the move by a random sign whenever it is used. This algorithm is not a Markov process, and therefore not genuine Monte Carlo, but it is able to discover precise and complicated enzymes that can be used to accelerate a legitimate Monte Carlo code.

The formula for health must be based on success in lowering the energy of the chain, but any reasonable implementation of this should work fine. We have chosen to multiply an individual's health by a factor $\lambda<1$ whenever it is tried, and add an amount proportional to the energy decrease of the chain if the new configuration is accepted. The population is updated periodically, and those that are unhealthy die off. We maintain a population of constant size; whenever one dies, two moves are selected randomly with probability proportional to their health, and a new individual is created as their vector sum or difference. One parent is occasionally zero-padded so the child can move more atoms.

We begin each run at high temperature, with the moves distributed randomly from 0 to 0.3 lattice spacings in length, then let the population evolve as the FK chain slowly cools. The population quickly adjusts to an appropriate length scale for the prevailing temperature, but with an excess of long moves for hopping atoms between wells, as shown in Figure 2. Upon cooling, the thermal distribution sharpens and the enzymes become separate, no longer just an anomalously long tail to the ordinary thermal peak.

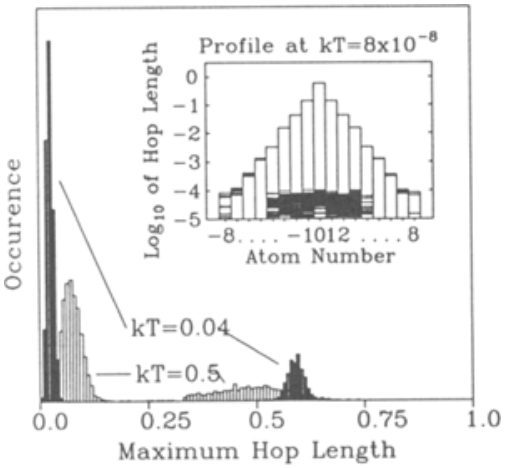

Figure 2: The population gradually develops precision, as shown by the histograms for $k T=0.5$ and 0.04 , and also complexity. By $k T=8 \times 10^{-8}$, each enzyme moves 16 atoms: number 0 by an amount of 0.5949 lattice spacings, and neighbors by smaller amounts; thermal moves are tiny and involve 6 to 8 atoms.

By the time the chain is cold enough that the first kind of defects have equilibrated out, the population discovers that the enzymes must move at least three atoms at once, moving one a large distance across the barrier and relaxing two neighbors. This is the needed move for equilibrating out a lower energy kind of defect. At still colder temperatures, the enzymes find that they need to move more and more atoms at once. If they fail to discover some necessary improvement, they will be unable to continue equilibrating defects out as the chain cools, and will become extinct, leaving only thermal moves in the population.

This algorithm is quite robust and not particularly sensitive to details in the algorithm, although some thought must be given to ensure that it generally encourages survival of the enzymes. For example, the relative scarcity of low en- 
ergy defects means that enzymes succeed infrequently at low temperatures; they simply cannot compete. We eliminate this bias by dividing the population into two classes, those that move at least one atom at least a third of a lattice spacing and those that do not, and multiplying the health of each individual by a normalization factor to promote equality between the classes. Of course, no amount of special treatment will help the enzymes if they fail to adapt to the changing environment and become unable to ever lower the energy of the chain.

If we cool slowly enough and set all the parameters to reasonable values, then long-range, fine-tuned enzymes usually develop that are capable of equilibrating out even very low energy defects. The inset to Figure 2 shows the 15-atom enzymes discovered by a run in which $\frac{K}{V}=\frac{1}{3}$. When we added this enzyme to a proper Monte Carlo code, we were able to equilibrate to a very low temperature and corresponding long correlation length, as shown in Figure 1.

In conclusion, we have found that diverging relaxation time scales prevent the FK model from equilibrating all the way to its zero-temperature ground state given finite cooling rate, and that much slower cooling results in equilibration to only slightly lower temperature. We have developed a method for accelerating relaxation in such a system in order to study the equilibrium behavior numerically. To do this, we use a modified Monte Carlo-like algorithm in which we keep track of the success of various moves, retaining and refining those that are especially useful for reducing the energy of the chain. We use the enzymes, or precisely coordinated motion of many atoms at once, that are discovered by the modified program to dramatically accelerate equilibration in a legitimate Monte Carlo code. The method should be especially useful in studying complicated models where the ground states and equilibrium properties cannot be deduced analytically.

This work was supported by Grant \#NSF-DMR-88-18558-A02 from the Cornell University Materials Science Center and an NSF graduate fellowship (SLS), and computer time was provided through the Cornell-IBM Joint Study on Computing for Scientific Research.

\section{REFERENCES}

1 The pinning transition is discussed in, e.g., S. N. Coppersmith, D. S. Fisher, Phys. Rev. B 28 (1983) 2566, and Braiman et. al., Phys. Rev. Lett. 65 (1990) 2398.

2 S. L. Shumway and J. P. Sethna, Phys. Rev. Lett. 67 (1991) 995.

${ }^{3}$ Michael Widom, Katherine J. Strandburg, and Robert H. Swendsen, Phys. Rev. Lett. 58 (1987) 706.

4 See, e.g., David E. Goldberg, Genetic Algorithms in Search, Optimization, and Machine Learning (Addison-Wesley, 1989). 